\title{
THE MINIMUM OF SMALL ENTIRE FUNCTIONS
}

\author{
P. C. FENTON
}

ABSTRACT. It is shown that if $f(z)$ is entire and satisfies $\overline{\lim } \log M(r, f) /(\log r)^{2}=\sigma$ $<\infty$ then for a sequence of $r \rightarrow \infty$

$$
m(r, f) / M(r, f)>\prod_{1}^{\infty}\left(\frac{1-\exp (-(2 k-1) / 4 \sigma)}{1+\exp (-(2 k-1) / 4 \sigma)}\right)^{2}+o(1) .
$$

This proves a long-standing conjecture of P. D. Barry.

1. Introduction. Suppose that $u(z)$ is subharmonic in the plane and let $B(r, u)=$ $\max _{|z|=r} u(z), A(r, u)=\inf _{|z|=r} u(z)$. It was proved by P. D. Barry [2, Theorem 8 and comments in \$7.4] that if

$$
\varlimsup_{r \rightarrow \infty} \frac{B(r, u)}{(\log r)^{2}}=\sigma<\infty
$$

then, on a sequence of $r \rightarrow \infty, A(r, u)>B(r, u)-\pi^{2} \sigma+o(1)$. The constant $\pi^{2} \sigma$ is known to be best possible over the class of subharmonic functions but Barry has shown [2, pp. 484-485] that a better constant is possible over the class of functions $u(z)=\log |f(z)|$, where $f$ is entire. He conjectured (see [2, p. 485]; also [1, p. 130]) that the right constant is $\log C$ rather than $\pi^{2} \sigma$, where

$$
C=\prod_{k=1}^{\infty}\left\{\frac{1+\exp \{-(2 k-1) / 4 \sigma\}}{1-\exp \{-(2 k-1) / 4 \sigma\}}\right\}^{2}
$$

an example [2, p. 484] shows that this would be best possible. The aim here is to prove Barry's conjecture.

THEOREM. Suppose that $f(z)$ is an entire function satisfying

$$
\varlimsup_{r \rightarrow \infty} \frac{\log M(r, f)}{(\log r)^{2}}=\sigma<\infty .
$$

Then given $\varepsilon>0$ there are certain arbitrarily large values of $r$ for which

$$
\log \frac{m(r, f)}{M(r, f)}>-\log C-\varepsilon,
$$

where $C$ is the number (1.2). Here $M(r, f)=\max _{|z|-r}|f(z)|$ and $m(r, f)=$ $\min _{|z|=r}|f(z)|$.

Received by the editors October 23, 1979 and, in revised form, April 11, 1980.

AMS (MOS) subject classifications (1970). Primary 30A64. 
2. Proof of the theorem: first part. We assume without loss of generality that the zeros of $f(z)$ are real and positive (so that in particular $f(0) \neq 0$ ), and write $\mu(t)$ for the number of zeros of $f(z)$ in $|z|<t$. (It should be mentioned that this counting function is usually devoted by $n(t)$ but since $n$ is used extensively later on as a subscript we make the change here to avoid subsequent confusion.) Then, (see [3, p. 271]), if $f(z)$ has no zeros on $|z|=r$,

$$
\log M(r, f)=r \int_{0}^{\infty} \frac{\mu(t)}{t(r+t)} d t, \quad \log m(r, f)=r \int_{0}^{\infty} \frac{\mu(t)}{t(r-t)} d t .
$$

The second of these integrals is to be understood as the principal value. Thus if $K$ is any real number, $\log m(r, f)>\log M(r, f)-K$ is exactly equivalent to (as is easily seen)

$$
\frac{1}{2} K>\int_{1}^{\infty} \frac{\mu(r x)-\mu(r / x)}{x^{2}-1} d x .
$$

Making the change of variable $r=e^{R}, x=e^{t}$, and writing $\nu(t)=\mu\left(e^{t}\right)$, becomes

$$
\frac{1}{2} K>\int_{0}^{\infty}(\nu(R+t)-\nu(R-t)) \frac{e^{t}}{e^{2 t}-1} d t
$$

which we rewrite as

$$
\frac{1}{2} K>\int_{0}^{\infty} \nu_{R}(t) \frac{e^{t}}{e^{2 t}-1} d t
$$

Here $\nu_{R}(t)=\nu(R+t)-\nu(R-t)$. If the zeros of $f(z)$ are $r_{1}<r_{2}<\cdots<r_{k}$ $<\ldots$, and $t_{k}=\log r_{k}$, then $\nu_{R}(t)$ measures the number of points $t_{k}$ in $[R-t, R$ $+t)$.

We aim to show that (2.3) holds for certain arbitrarily large values of $R$, for any fixed $K>\log C$; this will prove the theorem.

Using Jensen's theorem [3, p. 125] we deduce from (1.3) that $\mu(t)<$ $(2 \sigma+o(1)) \log t$ for arbitrarily large values of $t$, and from this it follows that, given $\varepsilon>0$, there are arbitrarily large integers $n$ such that

$$
\nu\left((1+\varepsilon) t_{n}\right)<(2 \sigma+2 \varepsilon) t_{n} .
$$

Let $m$ be any large positive integer, and let $n$ be greater than $m$ such that (2.4) holds. We form

$$
I_{p}=\min _{t_{p}<r<t_{p+1}} \int_{0}^{\infty} \nu_{r}(t) \frac{e^{t}}{e^{2 t}-1} d t
$$

for any $p$ such that both $m<p<n-1$ and $t_{p} \neq t_{p+1}$, and write $I_{m n}$ for $\min _{p} I_{p}$. We now rearrange the points $t_{k}$. First transfer all $t_{k}$ with $1 \leqslant k<m$ to the point $t_{m}$ and all $t_{k}$ satisfying $t_{n}<t_{k}<(1+\varepsilon) t_{n}$ to the point $t_{n}$. The effect of this change is to increase each of the minima $I_{p}$, and so increase $I_{m n}$, to $I_{m n}^{\prime}$ say. Simply ignoring the effect of the $t_{k}>(1+\varepsilon) t_{n}$ on the $I_{p}$ gives rise to an error no more than

$$
O\left(\int_{e t_{n}}^{\infty} t \frac{e^{t}}{e^{2 t}-1} d t\right)=O\left(t_{n} e^{-e t_{n}}\right)
$$


and produces a new minimum, $I_{m n}^{\prime \prime}$ say. Now place one point at each of $t_{m}-\tau k$ and $t_{n}+\tau k, k=1,2,3, \ldots$, where

$$
\tau=\frac{t_{n}-t_{m}}{\nu\left((1+\varepsilon) t_{n}\right)-1}
$$

The effect of this is to increase the minimum, to $I_{m n}^{\prime \prime \prime}$ say. Thus the $I_{m n}$ of the initial configuration of points $t_{k}$ is no larger than

$$
J_{m n}=\max I_{m n}^{\prime \prime \prime}+O\left(t_{n} e^{-t t_{n}}\right),
$$

where the maximum is taken in the following way: all points to the left of $t_{m}$ and to the right of $t_{n}$ must remain fixed; at least one point must remain at $t_{m}$ and at least one must remain at $t_{n}$. The remaining $\nu\left((1+\varepsilon) t_{n}\right)-2$ points are allowed to vary throughout the interval $\left[t_{m}, t_{n}\right]$. We stress that each $I_{m n}^{\prime \prime \prime}$ is the minimum (of minima of the form (2.5)) taken only over those $p$ for which $t_{p}$ and $t_{p+1}$ are successive points in $\left[t_{m}, t_{n}\right]$.

Now we shall clearly have (2.3) for certain arbitrarily large values of $R$ if it can be shown that for arbitrarily large values of $m$ there is an $n>m$ such that $I_{m n}<\frac{1}{2} K$. And this will certainly follow if, for certain arbitrarily large $m$, $\underline{\lim }_{n \rightarrow \infty} J_{m n}<\frac{1}{2} K$.

3. Proof of the theorem: second part. We show first that the maximum in (2.8) occurs when all the $\nu\left((1+\varepsilon) t_{n}\right)$ points in $\left[t_{m}, t_{n}\right]$ are distributed evenly, i.e. at a constant spacing $\tau$ given by (2.7).

In the configuration in which the maximum in (2.8) is attained, all the points must be separated and all the integrals $I_{p}$ must be the same. To see this it is necessary only to observe that the effect of pushing adjacent points radially the same (small) distance out from their midpoint is to increase all $I_{p}$ to the left of the smaller point and likewise all $I_{p}$ to the right of the larger point. Thus the effect of slightly separating two points which occur together, moving one slightly to the left and the other slightly to the right, is to increase $I_{m n}^{\prime \prime \prime}$; so points cannot occur together in the extremal configuration. Similarly, if one of the $I_{p}$, say $I_{p_{0}}$, is larger than all others, the effect of moving $t_{p_{0}}$ slightly to the left and $t_{p_{0}+1}$ slightly to the right is to increase all integrals $I_{p}, p \neq p_{0}$, and therefore to increase $I_{m n}^{\prime \prime \prime}$; so all integrals $I_{p}$ must be equal in the extremal configuration.

We show next that the only disposition of the points in $\left[t_{m}, t_{n}\right]$ in which all occur separately and all integrals $I_{p}$ are equal is the uniform distribution. This evidently follows from the following

LEMMA. Suppose that $a=s_{1}<s_{2}<s_{3}<\cdots<s_{N}=b$ and let

$$
\tau=(b-a) /(N-1) \text {. }
$$

Define

$$
s_{N+k}=b+k \tau, \quad k=1,2,3, \ldots
$$

and

$$
s_{1-k}=a-k \tau, \quad k=1,2,3, \ldots
$$


For $1<p<N-1$ define

$$
I_{p}=\min _{s_{p}<r<s_{p+1}} \int_{0}^{\infty} \nu_{r}(t) \frac{e^{t}}{e^{2 t}-1} d t
$$

If $I_{1}=I_{2}=\cdots=I_{N-1}$ then $s_{k}=a+\tau(k-1)$ for $1<k<N$.

The lemma is proved by induction. Suppose the lemma is true for a particular number $N_{0}(\geqslant 2)$ of points, no matter what the values of $a$ and $b$ (this is certainly true of 2 points), and suppose that $N=N_{0}+1$. There are three cases to consider.

(i) $s_{2}=s_{1}+\tau$. Then the result follows on applying the inductive hypothesis to the interval $\left[s_{2}, s_{N_{0}+1}\right.$, which contains $N_{0}$ distinct points.

(ii) $s_{2}<a+\tau$. We move $s_{2}$ to $a+\tau$; and if $s_{3}<s_{4}<\cdots<s_{k}$ are also in $\left(s_{2}, a+\tau\right]$ we shift them slightly to the right of $a+\tau$, preserving the order of all the points. The effect of this shifting is to increase every $I_{p}$ with $2<p<N_{0}$. (This is immediate for $N_{0}>p>k$. For $2<p<k, I_{p}$ will increase if the new positions of $s_{2}, \ldots, s_{k}$ are sufficiently close together.) Let us denote the new $I_{p}$ by $I_{p}^{\prime}$. (We shall add a prime at each change.) Since the effect on $I_{p}$ of shifting $s_{2}, \ldots, s_{k}$ declines as $p$ increases, we have $I_{k}^{\prime}>I_{k+1}^{\prime}>\cdots>I_{N_{0}}^{\prime}$. Moreover, we may fix the new positions of $s_{3}, \ldots, s_{k}$ in such a way that $I_{2}^{\prime}>I_{3}^{\prime}>\cdots>I_{k}^{\prime}>\cdots>I_{N_{0}}^{\prime}$; finally $I_{1}^{\prime}<I_{1}$.

Now consider the effect of the following shifting of the points. Shift $s_{N_{0}}$ far enough to the right that $I_{N_{0}}^{\prime \prime}=I_{N_{0}-1}^{\prime \prime}$. Then

$$
\begin{aligned}
& I_{N_{0}}^{\prime \prime}>I_{N_{0}}^{\prime}, \quad I_{j}^{\prime \prime}<I_{j}^{\prime}, \quad 1<j<N_{0}, \\
& I_{2}^{\prime \prime}>I_{3}^{\prime \prime}>\cdots>I_{N_{0}-1}^{\prime \prime}=I_{N_{0}}^{\prime \prime} .
\end{aligned}
$$

Having done this, shift $s_{N_{0}-1}$ far enough to the right that $I_{N_{0}-2}^{\prime \prime \prime}=I_{N_{0}-1}^{\prime \prime \prime}$. Then

$$
\begin{aligned}
& I_{N_{0}}^{\prime \prime \prime}>I_{N_{0}}^{\prime \prime}>I_{N_{0}}^{\prime}, \quad I_{N_{0}-1}^{\prime \prime \prime}>I_{N_{0}-1}^{\prime \prime}, \\
& I_{j}^{\prime \prime \prime}<I_{j}^{\prime \prime}, \quad 1<j<N_{0}-1, \\
& I_{2}^{\prime \prime \prime}>I_{3}^{\prime \prime \prime}>\cdots>I_{N_{0}-2}^{\prime \prime \prime}=I_{N_{0}-1}^{\prime \prime \prime}>I_{N_{0}}^{\prime \prime \prime} .
\end{aligned}
$$

Then repeat the process for $s_{N_{0}-2}, \ldots, s_{3}$ in turn. We might call the whole process a complete cycle. Let us call the minima at the end of a complete cycle $L_{1}, \ldots, L_{N_{0}}$. Then $L_{1}<I_{1}^{\prime}, L_{2}>L_{3}>\cdots>L_{N_{0}}, L_{2}<I_{2}^{\prime}$ and $L_{N_{0}}>I_{N_{0}}^{\prime}$. In other words, the effect of a complete cycle is to decrease the first minimum, to preserve the order of subsequent minima, and to decrease the second and increase the last minima. Thus a sequence of complete cycles will produce a situation in which all minima beyond the first have the same value, but a value which is strictly greater than the value of the first minimum. But from the inductive hypothesis the new $s_{2}, \ldots, s_{N_{0}+1}$ must be evenly spaced in $[a+\tau, b]$, so all minima must be the same, a contradiction. Hence $s_{2}<a+\tau$ cannot arise.

(iii) $s_{2}>a+\tau$. Again we move $s_{2}$ to $a+\tau$. The effect of this is to increase $I_{1}$, and to decrease every $I_{p}$ (to $I_{p}^{\prime}$ say) in such a way that $I_{2}^{\prime}<I_{3}^{\prime}<\cdots<I_{N_{0}}^{\prime}$. The argument of (ii) is now repeated but in the opposite direction: $s_{3}$ is the first point to be shifted, and it is shifted to the left. A contradiction again arises and the lemma is proved. 
4. Proof of the theorem: conclusion. The maximum of (2.8) occurs, we have shown, when the $\nu\left((1+\varepsilon) t_{n}\right)$ points in $\left[t_{m}, t_{n}\right]$ are evenly spaced, at a spacing $\tau$ given by (2.7). This gives

$$
\max I_{m n}^{\prime \prime \prime}=2 \sum_{k=1}^{\infty} \int_{\left(k-\frac{1}{2}\right) \tau}^{\infty} \frac{e^{t}}{e^{2 t}-1} d t=\log \left\{\prod_{k=1}^{\infty}\left(\frac{\exp ((2 k-1) \tau / 2)+1}{\exp ((2 k-1) \tau / 2)-1}\right)\right\} .
$$

Moreover when $n$ is large $\tau>1 /(2 \sigma+3 \varepsilon)$, and so for each fixed $m$

$$
\lim _{n \rightarrow \infty} J_{m n}<\log \left\{\prod_{k=1}^{\infty}\left(\frac{\exp ((2 k-1) /(4 \sigma+6 \varepsilon))+1}{\exp ((2 k-1) /(4 \sigma+6 \varepsilon))-1}\right)\right\} \text {. }
$$

It follows from this, together with the remark at the end of $\$ 2$, that (2.3) holds for arbitrarily large values of $R$ if $\frac{1}{2} K$ exceeds the right-hand side of (4.1). Since $\varepsilon$ is arbitrary the theorem is proved.

ADDED IN PROOF. The author is indebted to Professor W. K. Hayman for pointing out that Barry's conjecture was proved, using different methods, by A. A. Gol'dberg (Mat. Zametki 25 (1979), 835-844).

\section{REFERENCES}

1. J. M. Anderson, K. F. Barth and D. A. Brannan, Research problems in function theory, Bull. London Math. Soc. 9 (1977), 129-162.

2. P. D. Barry, The minimum modulus of small integral and subharmonic functions, Proc. London Math. Soc.(3) 12 (1962), 445-495.

3. E. C. Titchmarsh, Theory of functions, Oxford Univ. Press., New York, 1939.

Department of Mathematics, University of Otago, Dunedin, New Zealand 